\title{
Toward a High-Frequency Pulsed-Detonation Actuator
}

\author{
Andrew. D. Cutler ${ }^{*}$ \\ The George Washington University, Newport News, VA, 23602 \\ and \\ J. Philip Drummond ${ }^{\dagger}$ \\ NASA Langley Research Center, Hampton, VA, 23681
}

\begin{abstract}
This paper describes the continued development of an actuator, energized by pulsed detonations, that provides a pulsed jet suitable for flow control in high-speed applications. A high-speed valve, capable of delivering a pulsed stream of reactants-a mixture of $\mathrm{H}_{2}$ and air-at rates of up to 1500 pulses per second, has been constructed. The reactants burn in a resonant tube and the products exit the tube as a pulsed jet. High frequency pressure transducers have been used to monitor the pressure fluctuations in the device at various reactant injection frequencies, including both resonant and off-resonant conditions. Pulsed detonations have been demonstrated in the $\lambda / 4$ mode of an 8 inch long tube at $\sim 600 \mathrm{~Hz}$. The pulsed jet at the exit of the device has been observed using shadowgraph and an infrared camera.
\end{abstract}

\section{Introduction}

\section{A. Background}

B OUNDARY layer control can be broadly classified into passive and active control ${ }^{1}$. Common applications Binclude the control of boundary layer separation in external flows to maintain the effectiveness of flaps and other control surfaces, and internal flow boundary layer control associated with the operation of jet engine inlet, nozzle, and diffuser flows. Passive control includes the use of common devices such as vortex generator tabs ${ }^{2}$ to delay the onset of boundary layer separation.

Actuators of various types play an important role in active boundary layer control. A variety of different actuator devices have previously been investigated as flow control devices for both internal and external boundary layer applications. The operating principles for such devices are typically based on either mechanical deflection, mass injection, or the use of synthetic jets ${ }^{3}$. Mechanical actuator devices include conventional flap controls, as well as more recent MEMS flap devices. Mass injection actuators are characterized by a net mass flow through the actuator, and include combustion-driven jet actuators ${ }^{4}$. Synthetic jets are diaphragm devices that produce a pulsating jet for control of the boundary layer with no net mass injection ${ }^{5}$.

In high-speed applications, pulsed or synthetic jet actuators require both high impulse and high frequency. It is claimed $^{6}$ that dimensionless pulsation frequencies, defined as $\{$ frequency $\times$ streamwise length of separation $\} /$ free stream velocity , of $1-10$ are required for boundary-layer separation control (i.e., mitigation or removal of separation). This leads to the requirement for kilohertz level pulsation rates.

\section{B. Pulsed Combustion Actuator Concept}

Combustion jet actuators offer the promise of high impulse due to their utilization of the highly energetic combustion process. One such device, based on a deflagration (or subsonic wave) combustion process, is sparked to initiate combustion, and operates at a time scale between pulses very much greater than the time scales of wave propagation within the device ${ }^{7}$.

A new type of combustion-driven pulsed-jet actuator has been proposed ${ }^{8,9}$ that would (in concept) be capable of providing both high impulse and high frequency. The actuator is basically a small pulsejet engine ${ }^{10}$ in that it

\footnotetext{
* Professor, MAE Dep’t, 100 Old Oyster Point Road, Suite 200. Associate Fellow AIAA

† Senior Research Scientist, Hypersonic Air Breathing Propulsion Branch, MS 168. Associate Fellow AIAA
} 
operates in a resonant mode, taking advantage of wave propagation and reflection within the device to accelerate the combustion. Ideally, such a device, like the pulsejet engine, would be fueled passively: fuel and air would be periodically drawn into the device through aerodynamic or reed one-way valves as a consequence of the pressure variations associated with the wave motions. Typically, pressure fluctuations in pulsejets are not very high, perhaps a few 10's of psi. Since high impulse is sought for the actuator, detonation combustion, with its associated higher pressure fluctuation amplitudes, is desirable. Pulsed detonation engines (PDE) ${ }^{11}$ employ cyclical detonations in a tube, but have not been demonstrated at frequencies higher than $\sim 100 \mathrm{~Hz}$. One factor limiting the size and frequency of PDE's is the need to initiate a deflagration to detonation transition (DDT), which typically takes some significant length of tube. Because we seek to develop a device to operate at frequencies higher than has previously been demonstrated, the present conservative approach has been to actively fuel the device using a high-speed valve and to defer to the future the development of passive fueling methods. The fuel/oxidant considered is $\mathrm{H}_{2} /$ air, because of the high reactivity of these gases, and because of their ease of handling.

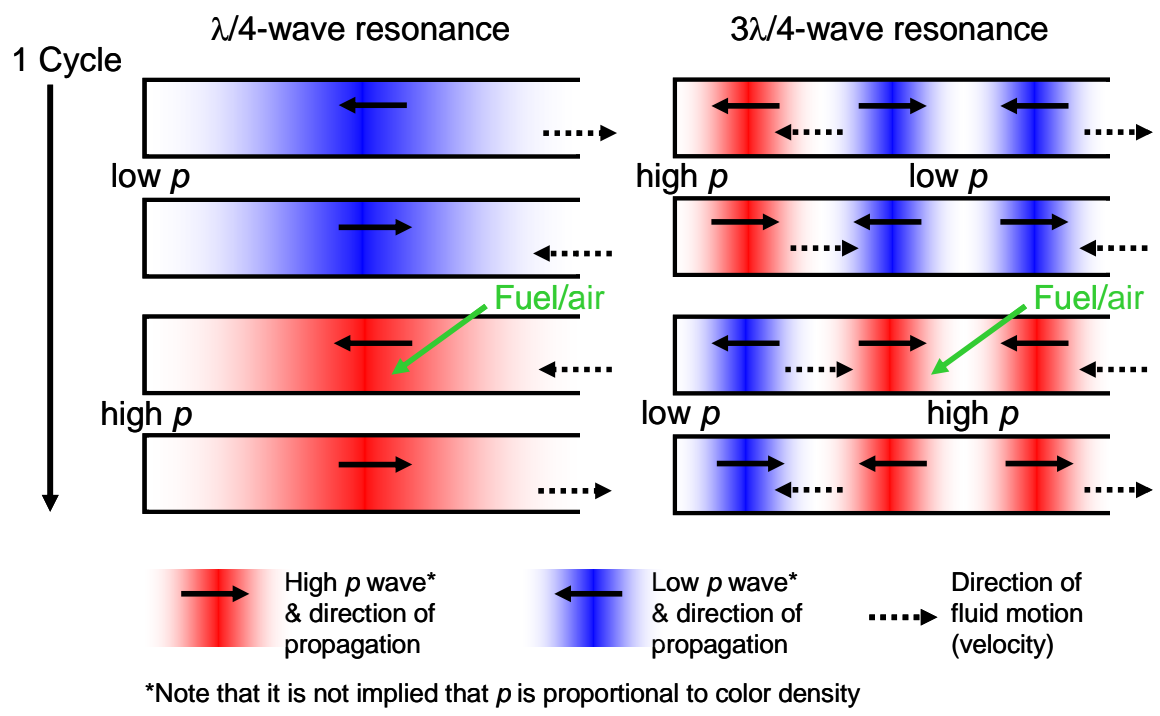

Figure 1. Resonant Detonation Actuator

The combustion actuator device under investigation is very simple, consisting of an inlet valve to periodically inject a charge of $\mathrm{H}_{2}$ /air mixture, a constant area tube, open at one end and closed at the other, in which the combustion takes place, and an igniter to initiate combustion. Products are discharged into the atmosphere (or flow field to be controlled) through the open end. This study is limited to the development of the actuator and not to its application, so discharge is into the laboratory atmosphere.

Multiple resonances of the flow in this tube can occur, as illustrated in Fig. 1. In this figure, two modes are

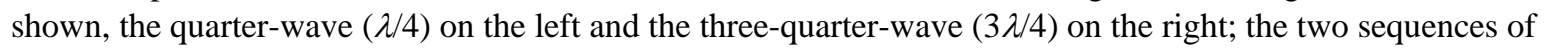
images represent the state of the flow in the tube at four equally-spaced times during a single cycle. The flow may be regarded as the superposition of right and left traveling pressure waves, represented by colored bars (red for high pressure and blue for low) with associated arrows representing direction of travel. Also shown are dashed arrows, representing the gas flow direction. Consider the $\lambda / 4$ sequence from top to bottom. An expansion wave initially travels left ( $1^{\text {st }}$ image $)$ and reflects from the closed end of the tube - the pressure in the tube is now at its lowest. The expansion wave travels right $\left(2^{\text {nd }}\right.$ image), and reflects from the open end of the tube as a compression - the velocity of the gas is now to the left. Reactants are injected into the tube from near the open end. The compression wave travels to the left ( $3^{\text {rd }}$ image) and reflects from the closed end as a compression - the pressure in the tube is now high. The reactants are ignited by contact with reaction products from the previous cycle together with the effects of compression and associated adiabatic heating, further raising the pressure. The compression wave (augmented by the effects of heat release) travels right $\left(4^{\text {th }}\right.$ image) and reflects from the open end as an expansion the velocity of the gas is now to the right, and reaction products are ejected from the tube. The $3 \lambda / 4$ resonance, illustrated by the right hand sequence, can be regarded as the superposition of larger number of traveling waves, and occurs at a higher frequency than the $\lambda / 4$.

Resonant frequencies for acoustic (small amplitude) waves may be computed from the following equation:

$$
f=\frac{a}{L}\left\{\frac{\text { Mode }}{\lambda}\right\}
$$


In this equation $a$ is the speed of sound, $L$ is the tube length, and Mode is $\lambda / 4,3 \lambda / 4$, etc. If the actuator is to be effective in producing a large amplitude pulsating jet, pressure fluctuation amplitudes will be large (detonation waves may occur) and "linear," acoustic assumptions will not hold, altering these frequencies.

An important feature of the actuator which distinguishes it from PDE's is that the reactants are not ignited by an ignitor at each injection cycle, but are ignited (as described above) by contact with the products of reaction from the previous cycle. Additionally, products are not displaced from the device by a non-reacting gas prior to injection of the next charge of reactants. This feature simplifies the fuel and air supply handling issues, an advantage in high frequency applications.

This work is a continuation of work reported in Cutler et al. ${ }^{9}$ In this previous work, pulsed combustion was demonstrated in several resonant modes of a 16 inch tube with 1.5 inch ID, including the $\lambda / 4$ and $3 \lambda / 4$, at frequencies up to $1400 \mathrm{~Hz}$. The flow in the tube was visualized by planar laser-induced fluorescence, and the flow at the exit by schlieren. One particularly interesting result was that at $\sim 285 \mathrm{~Hz}$, in the $\lambda / 4$ mode, peaky pressure waveforms were observed, suggestive of detonations (although the amplitudes were not those of a Chapman-Jouguet detonation wave). It was hypothesized in the reference that increasing the fraction of the length of tube filled by the reactants in this mode would lead to controlled periodic detonations, while reducing the length of the tube would lead to higher frequencies. The objective of the present work is to test this hypothesis.

\section{Experimental Method}

\section{A Actuator Model}

A new actuator model was fabricated for this work. It was of modular construction, with tube and fuel-air injector sections that could be screwed into one another. Many different configurations of the actuator could be constructed by using interchangeable components, and by changing their order of assembly. This paper is focused on one particular configuration, described in this section. Some (mainly negative) results for other configurations will be summarized in Section IIIB.

A line sectional drawing is shown in
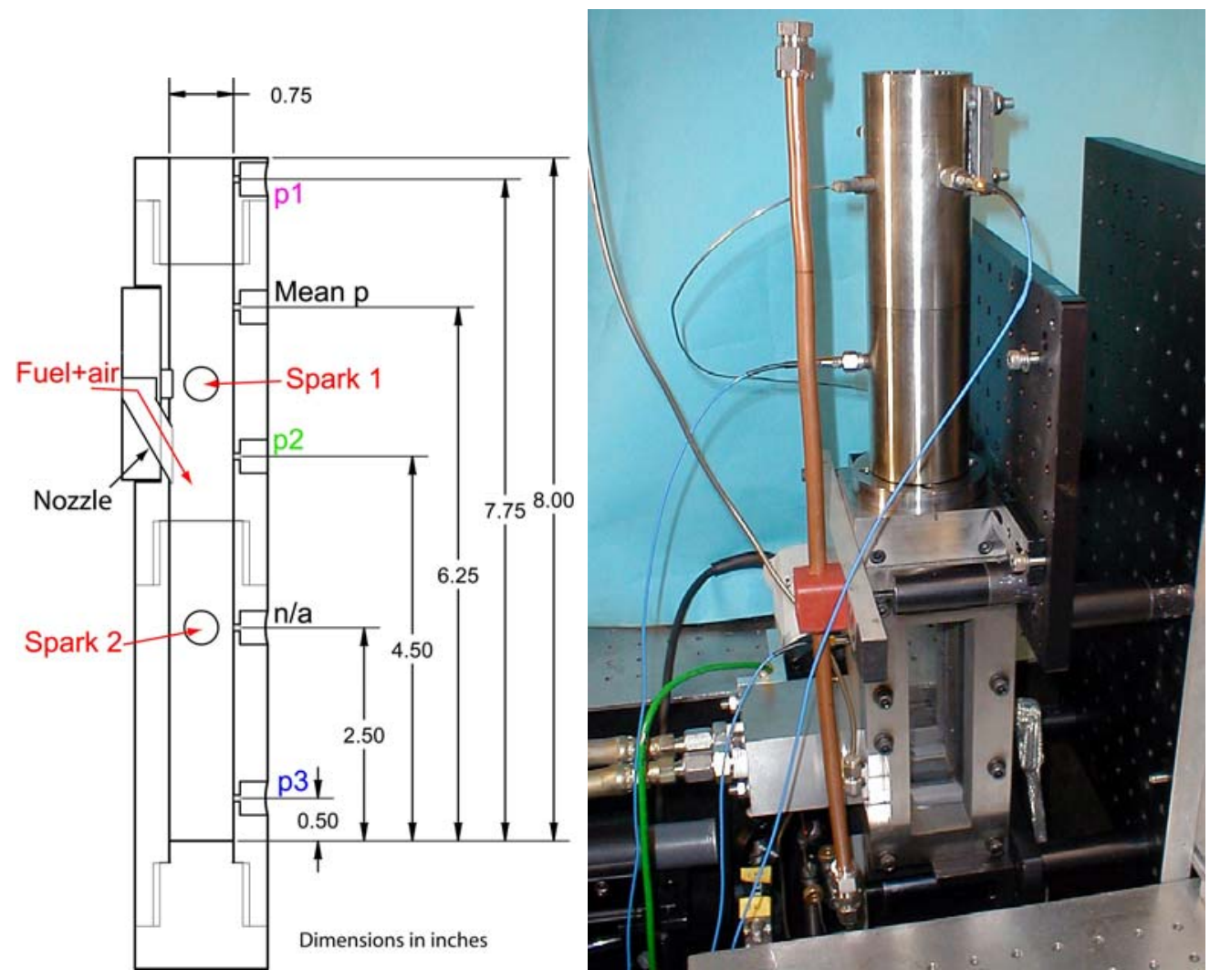

Figure 2. (a) Axial section of 8 inch actuator. (b) Photograph of mounted actuator assembly.

Fig. 2(a), and a photograph of the device is shown in Fig. 2(b). The combustor tube is 0.75 inches in diameter and 8 inches long, closed at the bottom and open at the top. Pulsed jets of $\mathrm{H}_{2}$ and air (or air- $\mathrm{O}_{2}$ mixture) from the valve (described below) are combined in the nozzle and the resulting flow enters the tube from the left 4.5 inches from the bottom at an angle of $30^{\circ}$ to the tube axis. The valve nozzle area minimum is 0.335 inches square. Two positions are provided for an automobile spark plug; the majority of the data reported use the plug at "Spark 2" only. To 
provide an initial ignition source, and to ensure reignition if the combustion becomes extinguished, the spark plug is fired continuously at $120 \mathrm{~Hz}$, independently of the injection process that occurs at frequencies from $100 \mathrm{~Hz}$ to 1500 $\mathrm{Hz}$.

The valve is the same as used in Ref. 9. A rendered view, with the valve body cut away in a plane through the $\mathrm{H}_{2}$ and air supply passages, is shown in Fig. 3(a). A detail of the rotating shaft is shown in Fig. 3(b). The valve consists of a rotating shaft, which is supported by high-speed bearings, with a hole (for $\mathrm{H}_{2}$ ) and a slot (for air). The shaft rotates in a housing containing passages for the $\mathrm{H}_{2}$ and air and a hole and slot slightly larger than the corresponding hole and slot in the shaft. When the holes and slots in the shaft and housing align-which occurs twice per shaft revolution- $\mathrm{H}_{2}$ and air pass through the valve and are combined in the small nozzle, prior to entering the combustor. The holes and slots are designed to provide a near stoichiometric mixture of hydrogen and air when the inlet supply pressures are equal.

A high-speed (up to $\sim 45,000 \mathrm{rpm}$ ) "universal” AC/DC motor turns the rotating shaft. This motor was originally manufactured to drive a range of interchangeable "homogenizer" tools; the valve is interchangeable with these tools, allowing quick disconnect of the motor. The motor speed is controlled by a DC motor controller, which can be set manually or by an external $0-10 \mathrm{~V}$ DC control voltage. This controller, however, cannot effectively maintain a constant speed. An electronic "proportional-integraldifferential," or PID, speed controller is available that receives the TTL pulses from the motor as feedback and outputs a control voltage to the motor controller: it is capable of maintaining speed within $1 \%$ of setpoint. Pressure taps are provided in the valve for monitoring both inlet hydrogen and inlet air pressures.

The valve and actuator were fabricated from stainless steel. They were not watercooled, although cooling air is blown across the exterior of the tube. However, they were designed to be massive, with thick walls, so run times of up to 15 seconds were possible before the temperatures became high enough to be of concern. After a run, blowing air through the valve and combustor cooled the assembly.

(a)

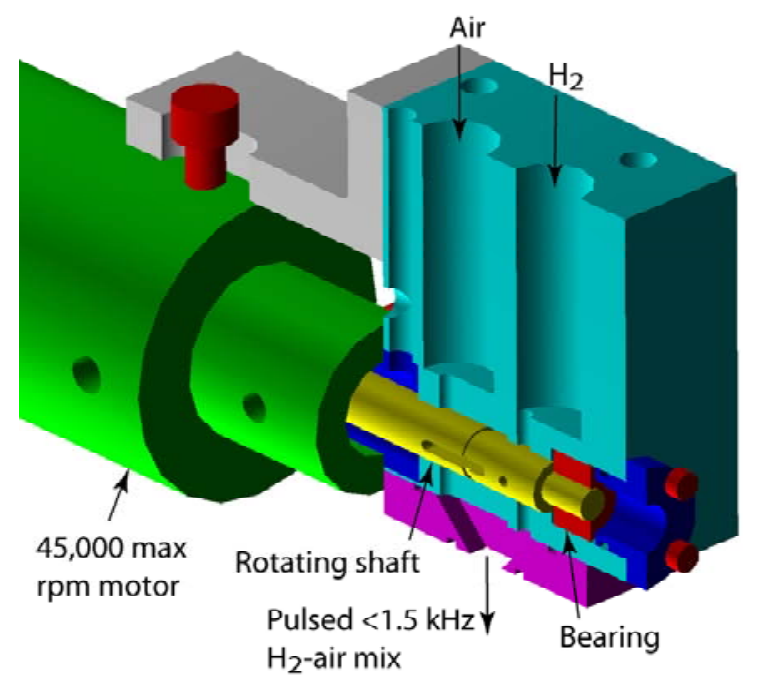

(b)

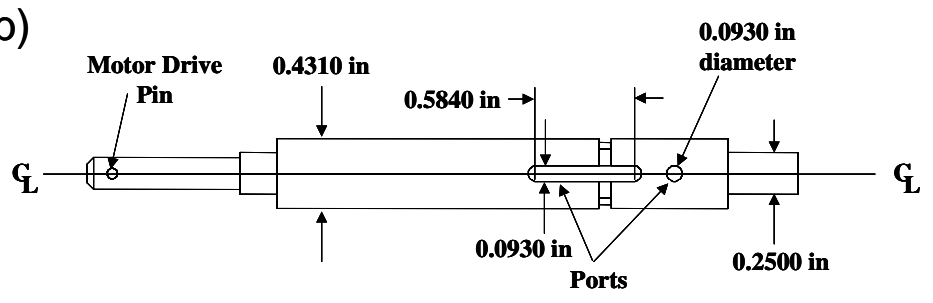

Figure 3. Actuator valve. (a) Partially cut-away view of valve \& motor. (b) Detail of rotating shaft.

\section{Miscellaneous Instrumentation}

Pressurized air and gaseous $\mathrm{H}_{2}$ fuel were supplied separately to the mixing valve. In certain experiments additional $\mathrm{O}_{2}$ was mixed with the air prior to entering the valve. Flows of $\mathrm{H}_{2}$, air and $\mathrm{O}_{2}$ were measured using Hastings mass flow meters of respectively 1000, 2500, and 300 standard liters per minute (10.6, 35.3, and 88.3 standard cubic feet per minute) full scale. Uncertainty in these measurements was $\sim 1 \%-2 \%$ of full scale.

Valve shaft position was measured by means of a helium-neon laser aimed at the rotating shaft, a retroreflective strip on the shaft, and a photodiode to detect reflected laser light. A custom-fabricated electronic package was used to convert the photodiode signal to a TTL pulse.

Taps were provided in the valve for monitoring both supply $\mathrm{H}_{2}$ and supply air mean pressures. Pressure taps were also provided in the combustion tube; these consisted of 0.076 inch deep by 0.076 inch diameter holes opening into a $10 \mathrm{~mm}$ threaded hole. Time-resolved pressure measurements were obtained within the combustion chamber at taps p1, p2, and p3, shown in Fig. 2(a). The measurements were by means of $0-250 \mathrm{lbf} / \mathrm{in}^{2}$ (psi) PCB-Piezotronics sensors model 113A21 (screwed into the threaded hole) and a 3-channel PCB-Piezotronics signal conditioning unit model 480A21. These gauges only provide AC-coupled (i.e., high-frequency-pass filtered) pressure. Separate measurements of mean pressure at a single tap were made using a strain gauge transducer. 
High speed pressure data ( 3 channels) and data from the valve shaft position photodiode ( 1 channel) were acquired with a digital oscilloscope, typically at a rate of 50,000 samples per second (per channel). A PC running a Labview program written for the purpose controlled the digital oscilloscope. Data were typically acquired for five seconds and then downloaded to the PC. The PC contained a National Instruments PCI-6025E multipurpose I/O board and preconditioning electronics that allowed simultaneous acquisition of $\mathrm{H}_{2}$, air and $\mathrm{O}_{2}$ flow rates from the mass flow meters and mean pressure data from the strain gauge pressure transducers. A control voltage could also be sent to the motor controller, ramping the motor speed up or down during the course of a run.

\section{Test Conditions}

During a typical run in which highfrequency pressure data were to be acquired, the valve motor was started, flow rates were established, combustion was initiated by the spark, data acquisition was initiated and simultaneously the valve motor was ramped either from low to high speed or from high to low speed. During tests in which flow visualization at the nozzle exit was performed the motor speed was held constant.

Several flow rate conditions are considered in this work, as summarized in Table 1 . The first two (no-combustion cases) have no flow of

\begin{tabular}{l|c|c|c|c|r|r|c} 
& \multicolumn{3}{|c|}{ Flow, scfpm } & \multicolumn{3}{c|}{ Flow, lbm/s } & \\
\hline Case & air & O2 & H2 & air & \multicolumn{1}{c|}{ O2 } & H2 & $\phi$ \\
\hline air-low & 26.5 & 0.00 & 0.0 & $3.56 \mathrm{E}-02$ & 0 & 0 & 0.000 \\
air-high & 53.0 & 0.00 & 0.0 & $7.12 \mathrm{E}-02$ & 0 & 0 & 0.000 \\
low & 26.5 & 0.00 & 10.6 & $3.56 \mathrm{E}-02$ & 0 & $9.91 \mathrm{E}-04$ & 0.952 \\
low/O2 & 23.8 & 2.65 & 10.6 & $3.21 \mathrm{E}-02$ & $3.93 \mathrm{E}-03$ & $9.91 \mathrm{E}-04$ & 0.692 \\
med & 39.7 & 0.00 & 15.9 & $5.34 \mathrm{E}-02$ & 0 & $1.49 \mathrm{E}-03$ & 0.952 \\
med/O2 & 35.7 & 3.99 & 15.9 & $4.81 \mathrm{E}-02$ & $5.93 \mathrm{E}-03$ & $1.49 \mathrm{E}-03$ & 0.691 \\
high & 53.0 & 0.00 & 21.2 & $7.12 \mathrm{E}-02$ & 0 & $1.98 \mathrm{E}-03$ & 0.952 \\
high/O2 & 47.7 & 5.30 & 21.2 & $6.41 \mathrm{E}-02$ & $7.87 \mathrm{E}-03$ & $1.98 \mathrm{E}-03$ & 0.692 \\
\hline
\end{tabular}

Table 1. Flow rates, inlet pressures, and tube pressures for the three combustion cases. $\mathrm{H}_{2}$ and two different rates of airflow. The remaining six (combusting) cases are grouped into three total volumetric flow rates (low, medium and high). At each total volumetric rate there are two cases, one with and one without supplemental $\mathrm{O}_{2}$. Supplemental $\mathrm{O}_{2}$ is $10 \%$ of the total of the air plus $\mathrm{O}_{2}$. Cases without supplemental $\mathrm{O}_{2}$ have close to stoichiometric proportions of $\mathrm{H}_{2}$ (the equivalence ratio $\phi$ is shown in the table). Since $\mathrm{H}_{2}$ is maintained constant, cases with added $\mathrm{O}_{2}$ have no potential for extra heat release, but have higher reactivity. Higher flow rates than the "high" cases were not tested due to a tendency for the bearings in the valve to fail due to the increased side-pressure load upon them.

\section{E. Optical Setups}

The flowfield at the exit of the actuator was visualized by simultaneously employing an infrared (IR) camera and a shadowgraph setup. The IR camera was a FLIR systems camera, framing at $180 \mathrm{~Hz}$ for the selected region of interest. The effective shuttering time of this camera in our experiment is not known precisely but, based on our results, must be significantly less than $0.3 \mathrm{~ms}$. When the camera was used to observe the tube pulsating at $\sim 350 \mathrm{~Hz}$, a frequency that "beats" with the $180 \mathrm{~Hz}$ camera rate, it produced sequences of images at small regularly-increasing phase-angles of the combustion cycle. The shadowgraph system employed a flashlamp of pulse length $\sim 1 \mu \mathrm{s}$, which froze the flow. However, the camera framed at $60 \mathrm{~Hz}$, and the flashlamp was not triggered in phase with the fuel injection, so results were at effectively random phase.

\section{F. Analysis of the Pressure-Time Histories}

Data from the digital oscilloscope were in the form of 4 channels of 250,000 samples each, acquired over 5 seconds: Channel 1 from the valve shaft position photodiode, Channels 2, 3, and 4 from pressure gauges p1, p2, and $\mathrm{p} 3$, respectively. Signals from the pressure gauges were converted from voltage to pressure (psi). For further reduction, the data were broken up into 0.1 sec segments of 5000 samples per channel.

For each $0.1 \mathrm{sec}$ segment, the photodiode data, which was a string of pulses of finite width, were processed to obtain a string of "delta" functions corresponding to the leading edge of each pulse, occurring once per shaft revolution. The injection frequency was found from the first maximum of the Fast Fourier Transform (FFT) of the processed Channel 1 signal, multiplied by two to convert shaft revolution frequency to valve opening frequency.

For each $0.1 \mathrm{sec}$ signal segment, the Channels 2, 3, and 4 (pressure) data were analyzed to obtain two separate results. The first result was the standard deviation (root-mean-square or RMS of the fluctuations). The second result was the one-cycle-delay correlation coefficient, found by taking the correlation coefficient of the data segment with itself shifted one reactant injection period in time. The correlation coefficient is exactly one if the signal is perfectly periodic at the injection rate and zero if perfectly random. 
Additionally, an artificial "signal" proportional to the effective opening area of the valve was constructed from the Channel 1 signal and knowledge of the valve geometry. A fixed phase offset was employed, found in a separate experiment in which data were acquired with one of the pressure transducers located at the exit of the valve.

\section{Experimental Results}

\section{A. Pressure Time History Results}

Pressure time history data have been reduced to obtain RMS pressure (psi) and one-cycle delay correlation coefficient, plotted as a function of injection frequency. Data were acquired for 8 cases, as summarized in Table 1. Reduced pressure results for various combustion cases are shown in Fig. 4; selected segments of pressure time history are shown in Figs. 5 and 6, calculated tube length filled by reactants is shown in Fig. 7, and results for air flow cases (no combustion) are shown in Fig. 8.

Consider Fig. 4. RMS pressure fluctuations for all gauges are high at low frequency and fall as the frequency increases (a, c). Fluctuations are highest for gauge p2 (near the middle of the tube) and lowest at p1 (near the exit), at all frequencies (a). For the high flow case, the correlation coefficients are high at frequencies less than about 800 $\mathrm{Hz}$ and again above about $1300 \mathrm{~Hz}$ (indicating periodic waveforms in these ranges), while they are low or negative in the intervening range (b). A correlation coefficient near zero indicates chaotic waveforms (or at least uncorrelated with fuel/air injection) while a negative coefficient indicates alternately positive and negative fluctuations of pressure at consecutive injection cycles. The effect of decreasing flow rate (from high to low) on gauge p2 is to decrease RMS pressure fluctuations in a manner roughly proportional to flow rate (c). The effect of
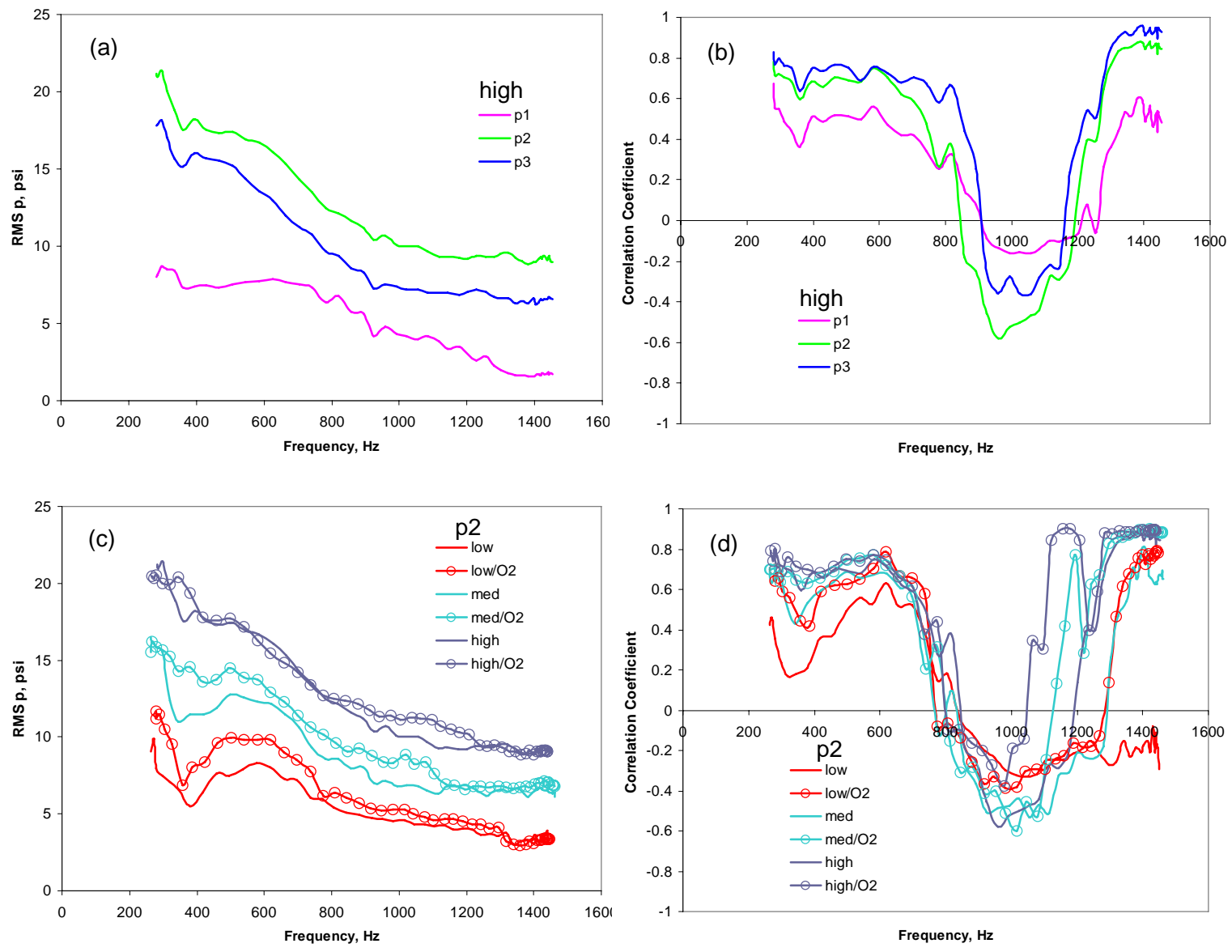

Figure 4. RMS pressure fluctuation and one-cycle-delay correlation coefficient as a function of injection frequency: (a, b) high flow case with gauges p1, p2, p3, and (c, d) various flow cases with gauge p2. 
decreasing flow rate on correlation coefficient is to reduce it in certain ranges, mostly noticeably at the highfrequency end where the coefficient becomes slightly negative at the low flow rate, but also in the vicinity of 350 $\mathrm{Hz}$. The effect of an increased $\mathrm{O}_{2}$ level, in general, is either negligible or to raise RMS fluctuation and correlation coefficient levels. At the high flow rate, the effect of $\mathrm{O}_{2}$ is negligible, except that in the $1000 \mathrm{~Hz}-1200 \mathrm{~Hz}$ range both the RMS fluctuation and correlation coefficient are raised. As flow rates are reduced the effect of $\mathrm{O}_{2}$ becomes progressively larger. Indeed, at the "low" rate and frequencies above $1300 \mathrm{~Hz}$ there is a large jump in correlation coefficient, from slightly negative to nearly 0.8 .

The lack of any effect of $\mathrm{O}_{2}$ for the high case (except for frequencies between $1000 \mathrm{~Hz}$ and $1200 \mathrm{~Hz}$ ), and the only small effect at some other conditions, is interesting. Added $\mathrm{O}_{2}$ should have the effect of increasing chemical kinetic rates. It should also compensate for incomplete mixing:- Cases without added $\mathrm{O}_{2}$ have an equivalence ratio near 1 (0.95), so almost every $\mathrm{O}_{2}$ molecule must find an $\mathrm{H}_{2}$ molecule to react with for all the $\mathrm{H}_{2}$ to react. On the other hand, cases with added $\mathrm{O}_{2}$ have an equivalence ratio of 0.69 , so only $69 \%$ of the $\mathrm{O}_{2}$ molecules need to find a partner, which requires less complete mixing. The results therefore suggest (for this case at least) complete mixing of the reactants. Complete mixing cannot be accomplished in the nozzle, so it must be accomplished after injection into the tube as a consequence of the processes taking place in the tube. Large pressure gradients (such as produced by detonations) acting at the interface of gases of different density (e.g., air and $\mathrm{H}_{2}$ or reactants and products) will accelerate the light gases relative to the heavy ones, increasing shear and mixing.
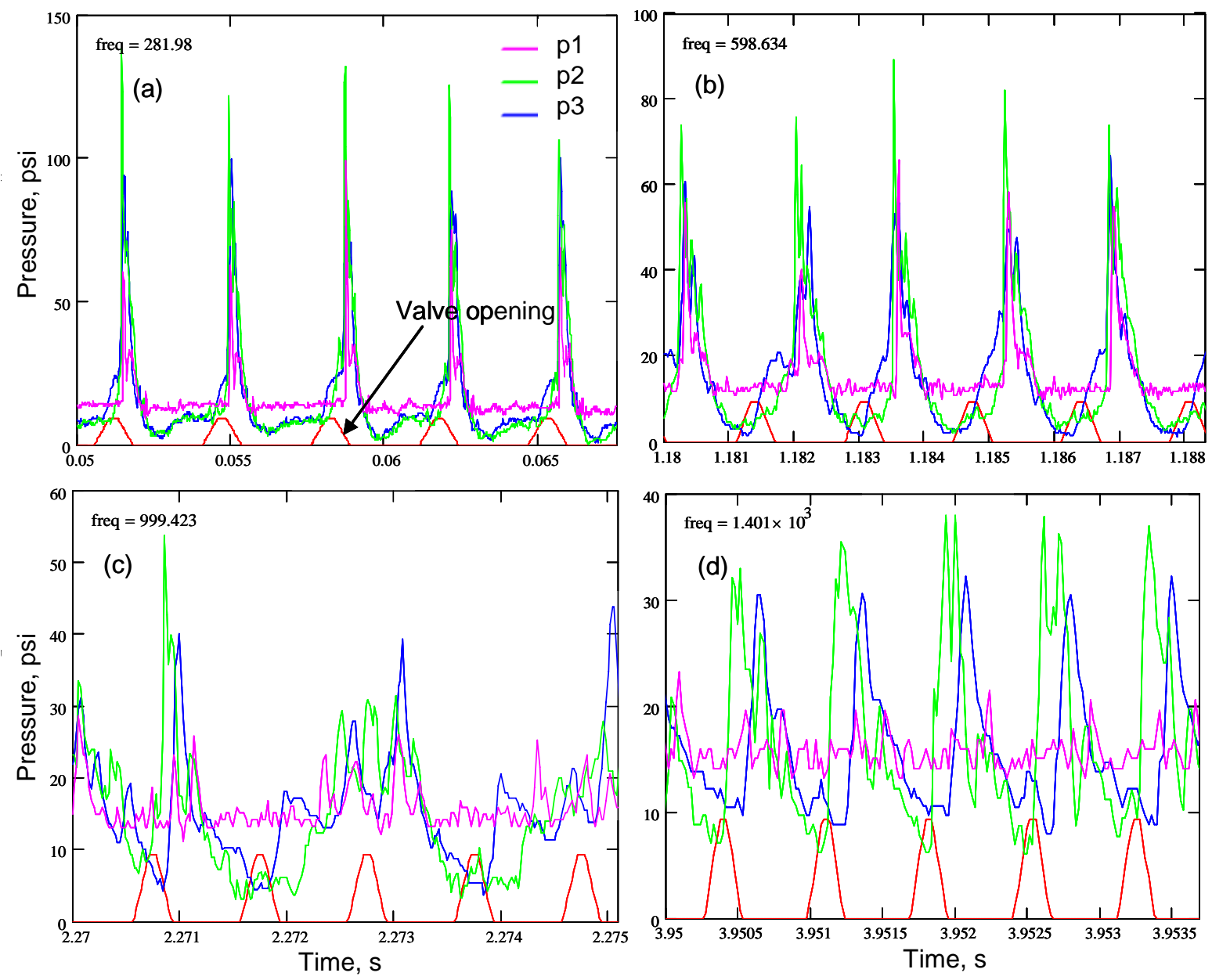

Figure 5. Four segments of pressure history for the high flow case: (a) $282 \mathrm{~Hz}$, (b) $599 \mathrm{~Hz}$, (c) $999 \mathrm{~Hz}$, and (d) $1401 \mathrm{~Hz}$ injection frequency.

Figure 5 shows four segments of pressure time history at various frequencies for the "high" flow case. Each segment contains 5 cycles of $\mathrm{H}_{2}$-air injection. The results from the pressure gauges, which indicate only pressure 
fluctuations not absolute pressure, have been added to the mean pressure measured at the single point in the tube indicated in Fig. 1. This may not be a very accurate way of getting absolute, time-dependent pressure since the mean pressure may depend on location in the tube. Also shown is the computed amplitude of the valve opening (i.e., the instantaneous injection rate) in arbitrary units.

At $282 \mathrm{~Hz}(\mathrm{a})$, the waveform is periodic at the injection frequency with a large-amplitude short-duration pressure spike in each cycle at all 3 gauges, suggestive of detonations. The peak of the spike is highest for $\mathrm{p} 2$ (between $100 \mathrm{psi}$ and $150 \mathrm{psi}$ ) and lowest for $\mathrm{p} 1$. The p1 signal has a spike superposed upon an essentially flat baseline, whereas for the p3 signal the spike is superposed upon a smoothly and periodically varying baseline. We believe this "baseline" is an approximately $\lambda / 4$ mode resonance at $\sim 564 \mathrm{~Hz}$, and that injection and detonation occurs every second cycle of this baseline resonance. Note that injection occurs shortly before combustion, in the rising pressure portion of this baseline.

At $599 \mathrm{~Hz}$ (b), the waveform is again periodic, with a spike occurring at each cycle of injection, but the peak amplitude is roughly half that at $282 \mathrm{~Hz}$. Again, the tube is operating at an approximately the $\lambda / 4$ mode resonance, but in this case with injection and detonation occurring at each cycle of this resonance. (The chaotic transition between injection and detonation occurring at each cycle of injection and occurring every second cycle can be seen in Fig. 4a and c as a dip in the RMS pressure fluctuations at $400 \mathrm{~Hz}$.)

At $999 \mathrm{~Hz}$ (c), the waveform is essentially chaotic, with varying amplitude oscillations (including some large spikes), indicating chaotic combustion with possibly intermittent detonations.

At $1401 \mathrm{~Hz}$ (d), the waveform is again periodic, with the pressure rise portion of the waveform broader (less "spikey") and the amplitude lower. The tube is probably operating at approximately the $3 \lambda / 4$ mode resonance.
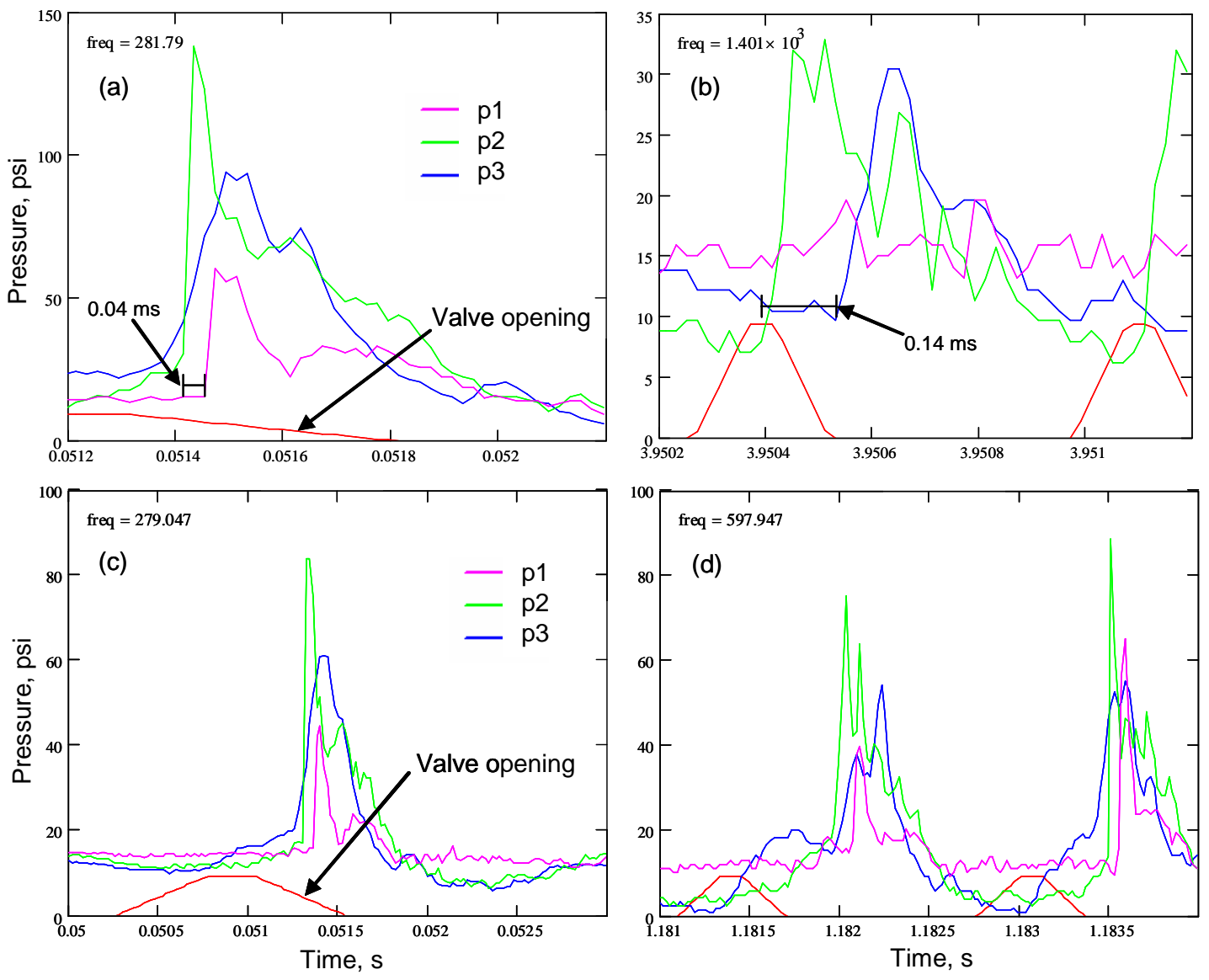

Figure 6. Four segments of pressure history: (a, b) $1 \mathrm{~ms}$ segments at $282 \mathrm{~Hz}$ and $1401 \mathrm{~Hz}$, same data as Fig. 5; (b, c) 3 ms segments at similar fill length, low/ $\mathrm{O}_{2}$ flow case at $279 \mathrm{~Hz}$ and high case at $598 \mathrm{~Hz}$,. 
Interestingly, the peak amplitude during the cycle seems to be roughly inversely proportional to the frequency, i.e., roughly proportional to the charge of $\mathrm{H}_{2}$-air burned in the cycle.

Figure 6 shows four segments of pressure time history over a smaller time scale. (a) and (b) are $1 \mathrm{~ms}$ time segments of the same data set as Fig. 5, the left is at $282 \mathrm{~Hz}$, the right at $1401 \mathrm{~Hz}$. Interestingly, the p2 and p3 waveforms (ignoring the baselines) are similar in width at the two frequencies. However, the peak at the leading edge of the pressure rise in gauge p2 is narrower, as well as being higher in amplitude at $282 \mathrm{~Hz}$. Note also that the pressure rise in this gauge leads the other gauges; the $0.04 \mathrm{~s}$ time between the rise of $\mathrm{p} 2$ and $\mathrm{p} 1$ (a) corresponds to a speed of $6800 \mathrm{ft} / \mathrm{s}$ while the $0.14 \mathrm{~ms}$ time between $\mathrm{p} 2$ and p3 (b) corresponds to about $2400 \mathrm{ft} / \mathrm{s}$. By comparison, the speed of the Chapman-Jouguet detonation wave for stoichiometric, atmospheric pressure $\mathrm{H}_{2}$-air is $\sim 6400 \mathrm{ft} / \mathrm{s}$. The consistency with the result at $282 \mathrm{~Hz}$ is striking, suggesting that the combustion is indeed a detonation, though it would be hard to imagine that the undoubtedly complex mixing and burning flow in this actuator would be the same in detail to the CJ wave. At $1401 \mathrm{~Hz}$, the combustion, though rapid and coupled to a compression wave, should probably not be described as a detonation. It is interesting to compare the peak pressure to the pressure of the CJ wave: 168 psia. At $282 \mathrm{~Hz}$, the peak pressure is 140 psia, only 20\% less than CJ, while it is much smaller at 1401 $\mathrm{Hz}$.

The fraction of the tube which is filled by reactants and is subsequently burned in an injection cycle is an important parameter that determines the nature of the combustion in the tube. The "fill length" is defined as the length of tube filled by reactants in one cycle, assuming standard temperature, and the fill fraction is the fill length divided by tube length. Figure 7 shows the fill length as a function of injection frequency for the various flow rates. One set of lines assumes a one atmosphere fill pressure. In practice, the tube is filled to above atmospheric pressure prior to combustion; the second set of lines therefore assumes fill to pressures determined by "eye" from the plots of pressure time history (by taking the maximum pressure prior to the sharp combustion pressure rise). Note that some of the tube will be filled by products from the previous cycle trapped at the end of the tube, which is not accounted for in

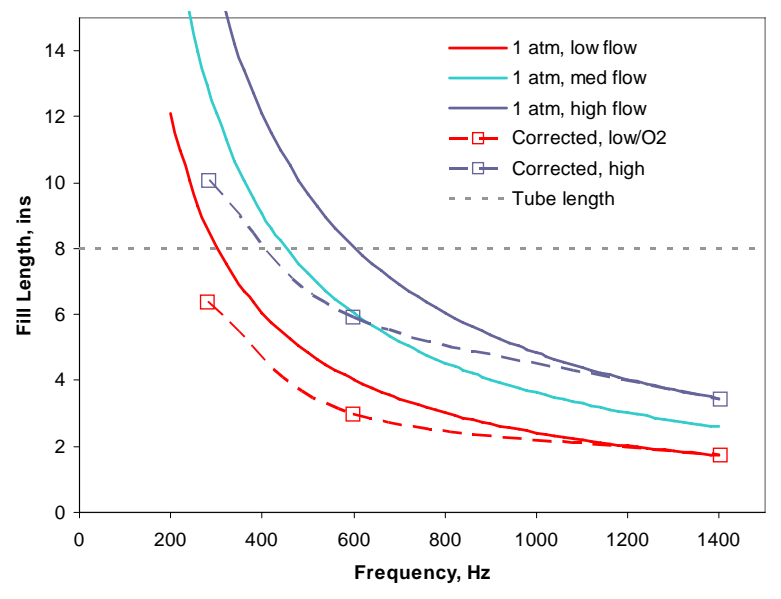

Figure 7. Variation in fill length with injection frequency. this plot. At $282 \mathrm{~Hz}$ in the high flow case the fill length exceeds the tube length so some reactants must vent from the tube prior to ignition. Figure 6(c, d) shows two 3 ms segments of segments of pressure time history at similar fill length: the "low $/ \mathrm{O}_{2}$ " flow case at $279 \mathrm{~Hz}$, and the high flow case at $598 \mathrm{~Hz}$. The waveforms for the two cases are remarkably similar to each other during the detonations; for all three gauges their differences are not much more than the random, cycle-to-cycle variation. A fill length of $\sim 1$ (i.e., the high flow, $282 \mathrm{~Hz}$ case) gives the best performance. We believe that a similar performance could be obtained at $\sim 600 \mathrm{~Hz}$ by increasing the flow rate (or reducing the tube diameter) to achieve a fill fraction near one, and at yet higher frequency by shortening the tube (to increase the $\lambda / 4$ resonant frequency) while maintaining the same flow rate and fill fraction.

Figure 8 shows results for injection of air only, i.e., no combustion. Reduced pressure results are shown as a function of injection frequency in (a) and (b). RMS pressure is shown in (a) for gauges p1, p2, and p3 at the high flow rate, and for gauge $\mathrm{p} 2$ at the low flow rate. Also shown are the theoretical resonant frequencies based on the acoustic wave speed in ambient temperature air: at $\lambda / 4$ it is $427 \mathrm{~Hz}$ and at $3 \lambda / 4$ it is $1280 \mathrm{~Hz}$. RMS pressure fluctuations for the p3 gauge peak around $5 \mathrm{psi}$ at $\sim 400 \mathrm{~Hz}$ and $\sim 1100 \mathrm{~Hz}$, roughly consistent with these modes (pressure fluctuations are much too high for the wave speeds to be acoustic, so the frequencies should not be expected to agree exactly). The effect of a $2 \times$ increase in flow rate (low to high) is to increase fluctuations, as expected, but by less than the increase in flow rate. The correlation coefficient is shown in (b) for gauges p1, p2, and $\mathrm{p} 3$ at the high flow rate. The correlation is high ( 0.9 or above) for gauges $\mathrm{p} 2$ and $\mathrm{p} 3$ at the resonances. Fivecycle segments of pressure time history are shown at $402 \mathrm{~Hz}$ in (c) and at $1107 \mathrm{~Hz}$ in (d). The waveforms at $402 \mathrm{~Hz}$ are periodic and qualitatively consistent in amplitude and shape with what that expected for an acoustic resonance at

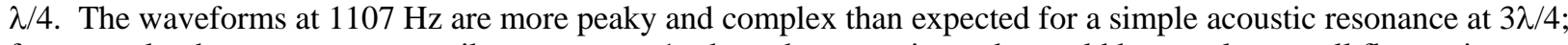
for example, there are pressure spikes at gauge $\mathrm{p} 1$ where the acoustic mode would have only a small fluctuation. 
However, the underlying $3 \lambda / 4$ mode is clear, with gauges $\mathrm{p} 1$ and $\mathrm{p} 2$ in phase with each other, while gauge $\mathrm{p} 3$ is $180^{\circ}$ out of phase with them.
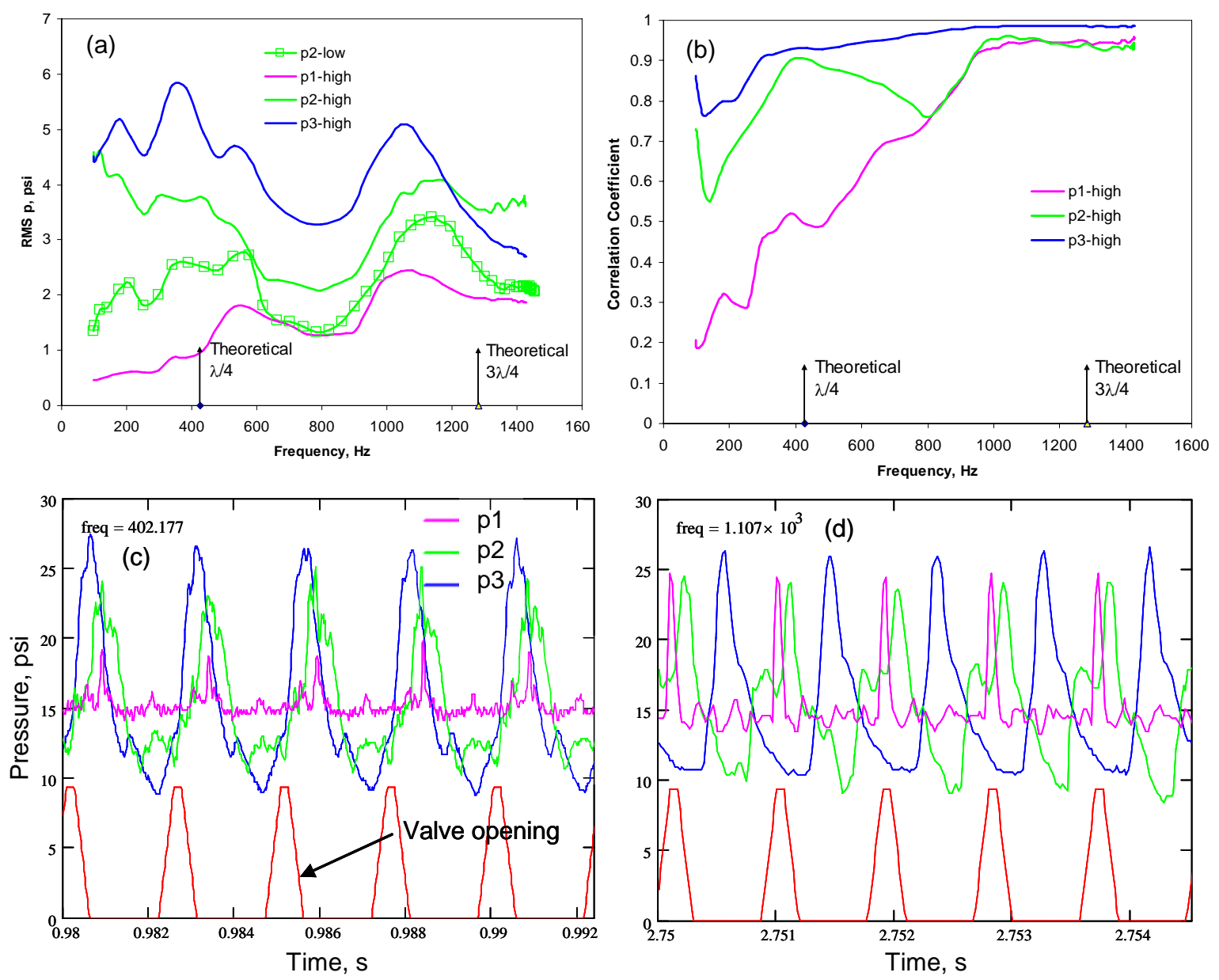

Figure 8. Pressure results for air flow (no combustion): (a) RMS pressure fluctuation, (b) one-cycle-delay correlation coefficient, (c) and (d) segments of pressure time history at $402 \mathrm{~Hz}$ and $1107 \mathrm{~Hz}$ respectively, near the $\lambda / 4$ and $3 \lambda / 4$ resonances.

Comparison of the previous results with the results for air flow shows the effects of combustion. RMS pressure fluctuations are much higher with combustion, as expected. However, the general trend in going to higher frequency is for the RMS fluctuations to decrease with combustion, while without combustion it is for them to remain roughly the same. This may be attributed to the more peaky (higher amplitude though narrower width) nature of the waveforms resulting from detonations, and the prevalence of detonations at the lower frequency. The

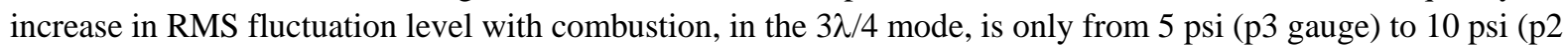
gauge). This increase seems small considering the $\sim 6.8 \times$ increase in volume of the flow as reactants convert to products (assuming an adiabatic, constant pressure process). However, the RMS pressure fluctuations were not proportional to volumetric rate in the air flow study. It is also possible that not all combustion heat release occurs in phase to enhance pressure fluctuations. Another effect of combustion is that the frequency of the resonances increases, for example from $\sim 400 \mathrm{~Hz}$ to $\sim 600 \mathrm{~Hz}$ for $\lambda / 4$. However, the increase is less than might be expected based on the increase in the speed of sound from air (1130 ft/s) to the combustion products ( $3200 \mathrm{ft} / \mathrm{s})$. This discrepancy may be due to the presence of unburned reactants in the tube during much of the cycle and, possibly, air entrained into the tube from the exit. It may also be due, in part, to the highly non-linear nature of the processes in the tube. 


\section{B. Role of Geometry and Spark}

As was discussed in Section IIA, the actuator was of a modular construction, permitting a number of configurations to be tested. The 8 inch tube illustrated in Fig. 2, the reference case, is only one of the latest configurations tested. These configurations may be subdivided into 3 groups that vary in the manner of injection into the tube.

In the first group, injection was from the bottom (closed end of the tube), i.e. at the tube axis, injecting parallel to the axis into the tube. This case was not successful because, with flows only as high as the "low" flow rate of Table 1, the bearings in the valve repeatedly failed. This is attributed to the impact of shock waves, through the valve nozzle, upon the valve shaft. Results obtained at one half the "low" flow rate were not encouraging, with chaotic waveforms, although results were also poor for the reference geometry at this flow rate.

In the second group, injection was from the side of the tube, perpendicular to the tube axis, near the bottom of the tube. In the third group, which includes the reference case, injection was from the side of the tube at $30^{\circ}$ to the tube axis. The third group had cases in which injection was from near the bottom of the tube, toward the exit, and cases with injection from near the exit of the tube, down, towards the closed end. Results were generally poor for cases with injection from near the bottom of the tube toward the exit. In all these cases, at the high flow rate, RMS pressure levels were generally lower than for the reference geometry while the waveforms were chaotic with intermittent detonations at low frequency, but somewhat better correlated waveforms at high frequency.

One case considered was identical to the reference case except that the tube was 10 inches long, instead of 8, lengthened by adding a tube section at the bottom. The results from this case were generally very similar to the reference case. The RMS pressure fluctuations were slightly lower in the lower frequency range and, predictably, the distribution of correlation coefficient with frequency was similar but shifted to lower frequency, indicating that the $\lambda / 4$ and the $3 \lambda / 4$ resonance frequencies were lower.

Another parameter is the location of the spark plug. Based on previous experiences (Ref. 9), it was believed that the location and role of the spark plug was unimportant once combustion was initiated in the tube. Tests of the effect of spark location were performed with the 10 inch tube just described at the high flow rate. The spark was provided from locations “Spark 1" and "Spark 2" (see Fig. 2), and a third location, "Spark 3", 0.5 inches from the bottom of the tube (not shown in Fig. 2 which is the 8 inch tube). The data of Section IIA are for location Spark 2. In location Spark 1, detonations occurred but were sporadic - Spark 1 is above where the reactants are injected into the tube. In location Spark 3, ignition of combustion could not be obtained at all - it is likely that products remain trapped at the bottom of the tube after the first cycle, preventing Spark 3 reaching fresh reactants. It was also found that, if the spark was turned off after initiating combustion in the tube, reaction would, in most cases, cease. Recall, the spark is fired at a fixed $120 \mathrm{~Hz}$, so individual detonations or combustion events occur multiple times between sparks. Thus the spark has some role in maintaining combustion but individual sparks are not required to initiate each combustion pulse. This matter requires further study.

While only a small fraction of all the possible permutations of our components (and spark locations) were studied, injection from near the exit of the tube down towards the closed end is the only one that provides periodic detonations in the $\lambda / 4$ resonance mode of the tube. The authors believe that this type of injection reinforces the natural motions of the $\lambda / 4$ mode, with the velocity of injection being aligned with and in phase with them.

\section{Flow Visualization Results}

The results of IR and shadowgraph visualization in the plume are shown in Fig. 9. These results are for the 10 inch tube, "high" flow rate. These results are for the convenient, $350 \mathrm{~Hz}$, injection frequency at which the $180 \mathrm{~Hz}$ framing rate of the IR camera beats with the injection frequency. For the 10 inch tube, this frequency is within the range of frequencies at which the $\lambda / 4$ mode resonance (with injection every cycle) takes place.

Figure 9(a) shows a sequence of IR images forming one typical cycle of injection. The IR radiation is believed to come from $\mathrm{H}_{2} \mathrm{O}$ vapor in the product gases. Black indicates cold gases and the color scale ranges through blue, orange and white at the highest temperatures. Images 1-3 and perhaps 4 show the ejection of hot gases from the actuator; in subsequent images ejection has probably ceased and the products are cooled through mixing with the ambient air. There is some indication of a diamond shock pattern in 3, and this type of pattern was seen in many other images at similar phase, indicating a supersonic ejection flow. Figure 9(b) shows selected typical shadowgraph images. Since these images were acquired at essentially random phase, they have been matched in phase, by subjective means, to several of the IR images. Image 1 clearly is at the beginning of an ejection; note the strong shockwave that leads the ejected gas. Images 5 and 9 seem to show a relatively slow moving turbulent plume (of products) at the nozzle exit. In 9, this plume tails to less than the diameter of the nozzle exit, indicating that ambient air and previously ejected products are being drawn back into the tube. There is no evidence in these 
images that reactants overfill the tube prior to combustion. This result is consistent with the calculated "corrected fill length" shown in Fig. 7, which is $\sim 9$ inches.

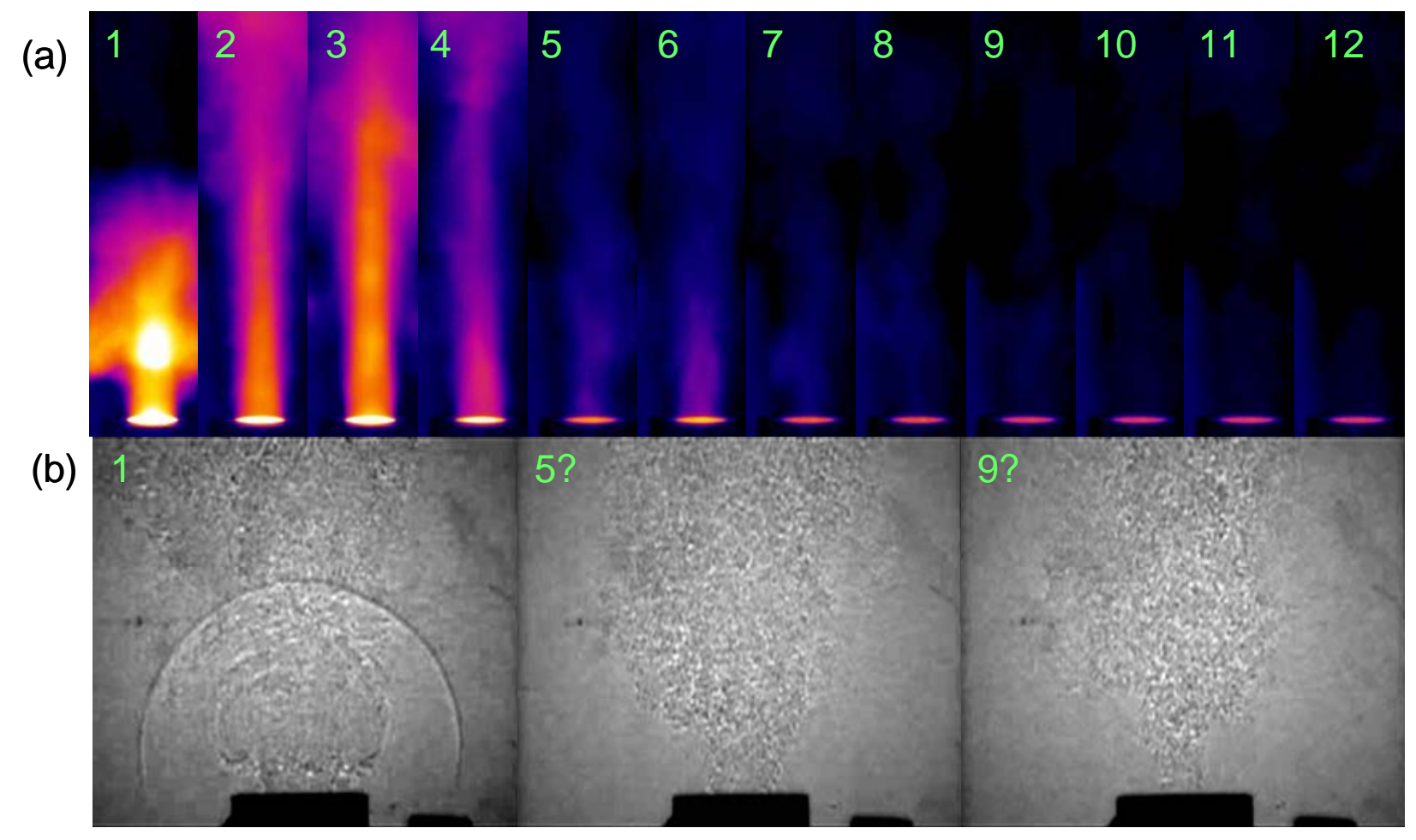

Figure 9. Flow visualization at the tube exit for a 10 inch tube, high flow case, pulsation at $350 \mathrm{~Hz}$; images at regular increments of phase over 1 cycle: (a) IR, (b) shadowgraph.

\section{Discussion}

The initial hypothesis of this work ${ }^{9}$, that detonations in a stoichiometric $\mathrm{H}_{2}$-air mixture could be accomplished in a short $\lambda / 4$ resonant tube if the fraction of the tube filled by reactants was sufficiently large, was proven. Indeed, it was shown that if the fill fraction approaches $\sim 1$ the peak pressure rise approaches $\sim 80 \%$ of that of a CJ detonation. In order to accomplish this high fill fraction, while maintaining cycle-to-cycle repeatable detonations, the reactants needed to be injected from near the exit toward the closed end of the tube, during the phase of the cycle when the air would normally be drawn into the tube from the outside. Detonation in the reactants is presumably initiated near the closed end of the tube and propagated towards the open end.

Detonation is difficult to initiate within a short distance; we cite the experiences of Lonneman and Cutler ${ }^{12}$ as typical. In these experiments, combustion was initiated by a spark in a $4 \mathrm{ft}$ long, 1.5 inch internal diameter constant area tube containing premixed $\mathrm{H}_{2}$, air and $\mathrm{O}_{2}$ in various ratios. It was found that, with a stoichiometric $\mathrm{H}_{2}$-air mixture at laboratory temperature, pressures from $1 \mathrm{~atm}$ up to 100 psia, and spark energy up to $15 \mathrm{~J}$, detonations could not be initiated before the end of the tube (although detonations could be initiated with $\mathrm{H}_{2}-\mathrm{O}_{2}$ mixtures). This has been the major practical difficulty in the development of PDE's. Possible reasons that detonations have been attained in the present work have been previously mentioned. By trapping combustion products from the previous cycle in the closed end of the tube, a source of high temperature and radical chemical species is available to initiate combustion. Also mentioned, wave motion in the $\lambda / 4$ wave mode raises the both pressure and temperature (via adiabatic work) at the closed end of the tube during the portion of the cycle immediately following injection of the reactants, accelerating combustion. Another, possible, contributing factor is the effect of wave curvature on the transition to detonation: if, for example, combustion is initiated at the exterior surface of a spherical volume of reactants, and propagates inwards, compression waves formed will strengthen as they approach the center by virtue of the geometry, accelerating the transition to detonation. It is speculated that, as the new charge of reactants enters the tube, curvature at the interface between reactants and surrounding hot products may, in this way, cause local detonating "spots" that then spread to the greater bulk of reactants. 


\section{Summary}

Experiments have been conducted to investigate pulsed combustion in a tube. The tube was 8 inches long, 0.75 inches in diameter, and fueled with pulses of $\mathrm{H}_{2}$ and air (or air $+\mathrm{O}_{2}$ ) in a stoichiometric ratio at up to $1400 \mathrm{~Hz}$. Periodic detonations were observed in the quarter-wave mode of resonance of the tube ( $\sim 60 \mathrm{~Hz}$ injection), and periodic combustion in the three-quarter wave ( $\sim 1400 \mathrm{~Hz}$ injection). To the author's knowledge, this is the first demonstration of periodic detonations in a tube at such high frequency. The fraction of the tube length filled by reactants was shown to be an important parameter in determining whether detonations occurred, with fractions as high as one producing the highest amplitude pressure fluctuations. Periodic combustion occurring at each injection cycle was accomplished only in cases in which the tube was filled by injecting reactants from near the tube exit towards the closed end. Experiments, in which some of the air was replaced by $\mathrm{O}_{2}$, while maintaining the same $\mathrm{H}_{2}$ rate, demonstrated that, in some cases at least, mixing and combustion of $\mathrm{H}_{2}$ and air was complete.

\section{Acknowledgments}

The first author would like to thank the NASA Langley Research Center for its support of this research through a Research Cooperative Agreement, NCC1-03011, and Technical Monitor Dr. A.H. Auslender. We would also like to thank Drs. Keith Grinstead and Daniel Bivolaru, and Stephen B. Jones for setting up and operating the shadowgraph and infrared camera systems used in this research, and providing other valuable assistance.

\section{References}

\footnotetext{
${ }^{1}$ Funk, R., Parekh, D., Crittenden, T. and Glezer, A., "Transient Separation Control using Pulse Combustion Actuation,” $1^{\text {st }}$ Flow Control conference, 24-26 June 2002, Paper\# AIAA 2002-3166.

${ }^{2}$ NASA Langley Research Center, Fact Sheet\# FS-2000-06-52-LaRC.

${ }^{3}$ Grossman, K. R., Cybyk, B. Z., and VanWie, D. M., "Sparkjet Actuators for Flow Control," 41 ${ }^{\text {st }}$ AIAA Aerospace Sciences Meeting \& Exhibit, 6-9 Jan. 2003, Paper\# AIAA-2003-0057.

${ }^{4}$ Crittenden, T. and Glezer, A., Funk, R., Parekh, D., “Combustion-Driven Jet Actuators for Flow Control,” $31^{\text {st }}$ AIAA Fluid Dynamics Conference \& Exhibit, 11-14 June 2001, Paper\# AIAA 2001-2768.

${ }^{5}$ Rinehart, C., McMichael, J. M., and Glezer, A., "Transitory Flow and force Development on a Body of Revolution Using Synthetic Jet Actuation,” Paper\# AIAA 2003-0618.

${ }^{6}$ Honohan, A.M., Amitay, M., Glezer, A., “Aerodynamic Control using Synthetic Jets,” AIAA Fluids 2000 Conference \& Exhibit, 19-22 June 2003, Paper\# AIAA-2000-2401.

${ }^{7}$ Kuo, K. K., Principles of Combustion, Wiley, 1986, pp. 231-283.

${ }^{8}$ B. T. Beck, A. D. Cutler, J. P. Drummond, S. B. Jones, “A Resonant Pulse Detonation Actuator for High-Speed Boundary Layer Separation Control,” 11th International Symposium On Flow Visualization, August 9-12, 2004, University of Notre Dame, Notre Dame, Indiana, USA, August 9-12, 2004.

${ }^{9}$ Cutler, A.D., Beck, B.T., Wilkes, J.A., Drummond, J.P., Alderfer, D.W., Danehy, P.M., "Development of a Pulsed Combustion Actuator For High-Speed Flow Control,” AIAA-2005-1084, 43rd AIAA Aerospace Sciences Meeting, Reno, NV, Jan 10-13, 2005.

${ }^{10}$ Diedrich, G. (translated by Kahane, A.), “The Aero-Resonator Power Plant of the V-1 Flying Bomb,” Project Squid TM Pr-4, Princeton University, 30 June 1948.

${ }^{11}$ Kailasanath, K., "Review of Propulsion Applications of Detonation Waves,” AIAA Journal, Vol. 38, No. 9, Sept 2000.

${ }^{12}$ Lonneman, P.J., Cutler, A.D., Development of an Actuator for Flow Control Utilizing Detonation, NASA/CR2004-213508, Dec. 2004.
} 